EUROPEAN JOURNAL OF PURE AND APPLIED MATHEMATICS

Vol. 12, No. 2, 2019, 499-505

ISSN 1307-5543 - www.ejpam.com

Published by New York Business Global

\title{
On Topologies Induced by Graphs Under Some Unary and Binary Operations
}

\author{
Caen Grace S. Nianga ${ }^{1, *}$, Sergio R. Canoy Jr. ${ }^{1}$ \\ 1 Department of Mathematics and Statistics, College of Science and Mathematics, \\ Center of Graph Theory, Algebra, and Analysis-PRISM, Mindanao State University-Iligan \\ Institute of Technology, 9200 Iligan City, Philippines
}

\begin{abstract}
Let $G=(V(G), E(G))$ be any simple undirected graph. The open hop neighborhood of $v \in V(G)$ is the set $N_{G}^{2}(v)=\left\{u \in V(G): d_{G}(u, v)=2\right\}$. Then $G$ induces a topology $\tau_{G}$ on $V(G)$ with base consisting of sets of the form $F_{G}^{2}[A]=V(G) \backslash N_{G}^{2}[A]$, where $N_{G}^{2}[A]=A \cup\left\{v \in V(G): N_{G}^{2}(v) \cap A \neq \emptyset\right\}$ and $A$ ranges over all subsets of $V(G)$. In this paper, we describe the topologies induced by the complement of a graph, the join, the corona, the composition and the Cartesian product of graphs.
\end{abstract}

2010 Mathematics Subject Classifications: 05C76

Key Words and Phrases: Join, Corona, Lexicographic product, Cartesian product, Open hop neighborhood

\section{Introduction}

Let $G=(V(G), V(H))$ be any simple undirected graph. The distance $d(u, v)$ between two vertices $u$ and $v$ in $G$ is the length of a shortest path joining $u$ and $v$. Let $v \in V(G)$. The neighborhood of $v$ is the set $N(v)$ consisting of all $u \in V(G)$ which are adjacent with $v$ and the closed neighborhood is $N[v]=N(v) \cup\{v\}$. For any $A \subseteq V(G)$, $N(A)=\{x: x a \in E(G)$ for some $a \in A\}$ is called the neighborhood of $A$ and $N[A]=N(A) \cup A$ is called the closed neighborhood of $A$. Moreover, for each $v \in V(G)$, the open hop neighborhood of $v$ is the set $N_{G}^{2}(v)=\left\{u \in V(G): d_{G}(u, v)=2\right\}$ and the closed hop neighborhood of $v$ is the set $N_{G}^{2}[v]=\{v\} \cup N_{G}^{2}(v)$. Also, for any $A \subseteq V(G)$, $N_{G}^{2}(A)=\left\{v \in V(G): N_{G}^{2}(v) \cap A \neq \emptyset\right\}$ is called the open hop neighborhood of $A$ and the set $N_{G}^{2}[A]=A \cup N_{G}^{2}(A)$ is the called closed hop neighborhood of $A$. Denote by $F_{G}^{2}[A]$ the complement of $N_{G}^{2}[A]$, i.e., $F_{G}^{2}[A]=V(G) \backslash N_{G}^{2}[A]$.

In 1983, Diesto and Gervacio in [5] proved that given a simple graph $G=(V(G), E(G))$, $G$ induces a topology on $V(G)$, denoted by $\tau_{G}$, with base consisting of sets of the form

${ }^{*}$ Corresponding author.

DOI: https://doi.org/10.29020/nybg.ejpam.v12i2.3421

Email addresses: caengrace1997@gmail.com (C. Nianga), sergio.canoy@g.msuiit.edu.ph (S. Canoy Jr.) 
$F_{G}(A)=V(G) \backslash N_{G}(A)$, where $N_{G}(A)=A \cup\{x: x a \in E$ for some $a \in A\}$ and $A$ ranges over all subsets of $V(G)$. Their construction was further investigated in [2], [3] and [6]. In particular, Canoy and Lemence in [2] described the topologies induced by the complement of a graph, the join of graphs, composition and Cartesian product of graphs.

In [1], Canoy and Gimeno presented another way of constructing a topology $\tau_{G}$ from a connected graph $G$ by considering the family $\Omega(G)=\left\{F_{G}^{2}[A]: A \subseteq V(G)\right\}$ where $F_{G}^{2}[A]=\left\{x \in V(G): x \notin A\right.$ and $d_{G}(x, a) \neq 2$ for all $\left.a \in A\right\}$. They showed that this family is a base for some topology $\tau_{G}$ on $V(G)$. This construction is also studied by Nianga et al, in [4] for any graph $G$. It is also shown that the family $\mathcal{B}_{G}=\left\{F_{G}^{2}[A]: A \subseteq V(G)\right\}$ and $\mathcal{S}_{G}=\left\{F_{G}^{2}[v]: v \in V(G)\right\}$ are, respectively, base and subbase for the topology $\tau_{G}$ on $V(G)$.

Concepts on Graph Theory and Topology are taken from [7] and [8], respectively.

\section{Results}

Definition 1. The complement of graph $G$, denoted by $\bar{G}$ is the graph with $V(G)=V(\bar{G})$ and $u v \in E(\bar{G})$ if and only if $u v \notin E(G)$, where $u, v \in V(G)=V(\bar{G})$.

Theorem 1. Let $G$ be any graph and $\bar{G}$ its complement. Then for each $v \in V(G)$,

$$
F_{\bar{G}}^{2}[v]=\left\{\begin{array}{cl}
F_{G}[v] \cup\left[\bigcap_{u \in F_{G}[v]} N_{G}(u)\right], & \text { if } F_{G}[v] \neq \emptyset \\
N_{G}(v), & \text { if } F_{G}[v]=\emptyset .
\end{array}\right.
$$

Proof. Let $G$ be any graph and $\bar{G}$ its complement. Let $v \in V(G)$ and set $A=\cap_{u \in F_{G}[v]} N_{G}(u)$. Suppose $F_{G}[v]=\emptyset$. Then $N_{G}(v)=V(G) \backslash\{v\}$. Hence, $v$ is an isolated vertex in $\bar{G}$. Thus, $F_{\bar{G}}^{2}[v]=N_{G}(v)$. Suppose $F_{G}[v] \neq \emptyset$. Let $u \in F_{G}[v]$. Then $u \neq v$ and $u \notin N_{G}(v)$. Hence, $u \neq v$ and $u \in N_{\bar{G}}(v)$. Thus, $u \in F_{\bar{G}}^{2}[v]$. Next, let $w \in A$. Then $w \in N_{G}(u)$ for all $u \in F_{G}[v]$. Since $u \notin N_{G}(v)$, it follows that $w \neq v$. Also, $w \notin N_{\bar{G}}(u)$ for all $u \in N_{\bar{G}}(v)$. It implies that $d_{\bar{G}}(w, v) \neq 2$. Hence, $w \in F_{\bar{G}}^{2}[v]$. Consequently, $F_{G}[v] \cup\left[\cap_{u \in F_{G}[v]} N_{G}(u)\right] \subseteq F_{\bar{G}}^{2}[v]$. Next, let $x \in F_{\bar{G}}^{2}[v]$. Then $x \neq v$ and $x \notin N_{\bar{G}}^{2}(v)$. If $x \in F_{G}[v]$, then we are done. Suppose $x \notin F_{G}[v]$. Then $x \in N_{G}(v)$. Suppose further that there exists $u \in F_{G}[v]$ such that $x \notin N_{G}(u)$. Thus, $u \in N_{\bar{G}}(v)$ and $x \in N_{\bar{G}}(u)$. Also, since $x \in N_{G}(v), x \notin N_{\bar{G}}(v)$. Thus, $d_{\bar{G}}(x, v)=2$, that is, $x \in N_{\bar{G}}^{2}(v)$, a contradiction. Therefore, $x \in N_{G}(u)$ for all $u \in F_{G}[v]$. This shows that $x \in A$. Accordingly, $F_{\bar{G}}^{2}[v] \subseteq F_{G}[v] \cup\left[\cap_{u \in F_{G}[v]} N_{G}(u)\right]$. This establishes the desired equality.

Theorem 2. Let $G$ be any graph and $\bar{G}$ its complement. If $v$ is an isolated vertex of $G$ (or of $\bar{G}$ ), then $\{v\} \in \tau_{G} \cap \tau_{\bar{G}}$.

Proof. Suppose $v$ is an isolated vertex of $G$ (or of $\bar{G}$ ). Then $\{v\}=F_{G}^{2}[V(G) \backslash\{v\}]=F_{G}^{2}[V(\bar{G}) \backslash\{v\}]$ and so, $\{v\} \in \mathcal{B}_{G}$ and $\{v\} \in \mathcal{B}_{\bar{G}}$. Thus, $\{v\} \in \tau_{G}$ and $\{v\} \in \tau_{\bar{G}}$. Therefore, $\{v\} \in \tau_{G} \cap \tau_{\bar{G}}$. 
Remark 1. The converse of theorem 17 is not true.

Consider $G=P_{5}=[a, b, c, d, e]$. Then $\{e\}=F_{G}^{2}[a, b]$ and $\{e\}=F_{\bar{G}}[a, c]$. However, $e$ is not an isolated vertex of $G$ nor of $\bar{G}$.

Definition 2. The join $G_{1}+G_{2}$ of graphs $G_{1}$ and $G_{2}$ is the graph $G$ with $V(G)=V\left(G_{1}\right) \cup V\left(G_{2}\right)$ and

$$
E(G)=E\left(G_{1}\right) \cup E\left(G_{2}\right) \cup\left\{u v: u \in V\left(G_{1}\right) \text { and } \mathrm{v} \in \mathrm{V}\left(\mathrm{G}_{2}\right)\right\} .
$$

Theorem 3. Let $G=(V(G), E(G))$ and $H=(V(H), E(H))$ be graphs and let $\emptyset \neq A \subseteq V(G)$ and $\emptyset \neq B \subseteq V(H)$. Then

(i) $F_{G+H}^{2}[A]=V(H) \cup\left[\cap_{a \in A} N_{G}(a)\right]$;

(ii) $F_{G+H}^{2}[B]=V(G) \cup\left[\cap_{b \in B} N_{H}(b)\right]$ and

(iii) $F_{G}^{2}[\emptyset]=V(G) \cup V(H)$.

Proof. Let $G=(V(G), E(G))$ and $H=(V(H), E(H))$ be graphs. Let $\emptyset \neq A \subseteq V(G)$ and $\emptyset \neq B \subseteq V(H)$.

(i) Note that

$$
N_{G+H}^{2}[A]=A \cup\left\{v \in V(G+H): d_{G+H}(v, a)=2 \text { for some } a \in A\right\} .
$$

Since $V(H) \subseteq N_{G+H}(A)$,

$$
\begin{aligned}
N_{G+H}^{2}[A] & =A \cup\left\{v \in V(G): d_{G+H}(v, a)=2 \text { for some } a \in A\right\} \\
& =A \cup\left\{v \in V(G): d_{G}(v, a) \neq 1 \text { for some } a \in A\right\} .
\end{aligned}
$$

Hence,

$$
F_{G+H}^{2}[A]=V(H) \cup\left[\cap_{a \in A} N_{G}(a)\right] .
$$

(ii) Similarly,

$$
F_{G+H}^{2}[B]=V(G) \cup\left[\cap_{b \in B} N_{H}(b)\right] .
$$

(iii) Clearly, $F_{G+H}^{2}[\emptyset]=V(G) \cup V(H)$.

Remark 2. Let $G$ be any graph and let $A_{1}, A_{2} \subseteq V(G)$. Then

$$
N_{G}^{2}\left[A_{1} \cup A_{2}\right]=N_{G}^{2}\left[A_{1}\right] \cup N_{G}^{2}\left[A_{2}\right] .
$$

Theorem 4. Let $G=(V(G), E(G))$ and $H=(V(H), E(H))$ be graphs. Then for any $A \subseteq V(G+H)$ such that $A \cap V(G)=A_{G} \neq \emptyset$ and $A \cap V(H)=A_{H} \neq \emptyset$,

$$
F_{G+H}^{2}[A]=F_{G+H}^{2}\left[A_{G}\right] \cap F_{G+H}^{2}\left[A_{H}\right] .
$$


Proof. Let $A \subseteq V(G+H)$. Suppose $A \cap V(G)=A_{G} \neq \emptyset$ and $A \cap V(H)=A_{H} \neq \emptyset$. Then $x \in F_{G+H}^{2}[A]$ if and only if $x \notin N_{G+H}^{2}[A]$. By Remark 2, $x \in F_{G+H}^{2}[A]$ if and only if $x \in F_{G+H}^{2}\left[A_{G}\right] \cap F_{G+H}^{2}\left[A_{H}\right]$.

The next theorem follows from Theorem $3(i)$ and $(i i)$.

Corollary 1. Let $G=(V(G), E(G))$ and $H=(V(H), E(H))$ be graphs. Then for any $v \in V(G) \cup V(H)$,

$$
F_{G+H}^{2}[v]= \begin{cases}V(H) \cup N_{G}(v), & \text { if } v \in V(G) \\ V(G) \cup N_{G}(v), & \text { if } v \in V(H) .\end{cases}
$$

Definition 3. The corona $G \circ H$ of graphs $G$ and $H$ is the graph obtained by taking one copy of $G$ and $|V(G)|$ copies $H$ and then forming the sum $\langle v\rangle+H^{v}=v+H^{v}$ for each $v \in V(G)$, where $H^{v}$ is a copy of $H$ corresponding to the vertex $v$.

Theorem 5. Let $G=(V(G), E(G))$ and $H=(V(H), E(H))$ be graphs. Then for any $a \in V(G \circ H)$,

$$
F_{G \circ H}^{2}[a]=\left\{\begin{array}{cl}
F_{G}^{2}[a] \cup\left[\bigcup_{v \in V(G) \backslash N_{G}(a)} V\left(H^{v}\right)\right], & \text { if } a \in V(G) \\
N_{H^{w}}(a) \cup\left[V(G) \backslash N_{G}(w)\right] \cup\left[\bigcup_{v \in V(G) \backslash\{w\}} V\left(H^{v}\right)\right], & \text { if } a \in V\left(H^{w}\right)
\end{array}\right.
$$

Proof. Let $x \in F_{G \circ H}^{2}[a]$. Then $x \neq a$ and $x \notin N_{G \circ H}^{2}(a)$. Consider the following cases:

Case 1. Suppose $a \in V(G)$. If $x \in V(G)$, then $x \notin N_{G}^{2}(a)$ since $x \notin N_{G \circ H}^{2}(a)$. Hence, $x \in F_{G}^{2}[a]$. Suppose $x \notin V(G)$. Let $u \in V(G)$ such that $x \in V\left(H^{u}\right)$. If $u=a$, then $x \in V\left(H^{u}\right)$ and $u \in V(G) \backslash N_{G}(a)$. Suppose $u \neq a$. Since $x \notin N_{G}^{2}(a)$ and $d_{G \circ H}(a, y)=2$ for all $y \in V\left(H^{z}\right)$ with $z \in N_{G}(a)$, it follows that $u \in V(G) \backslash N_{G}(a)$. Thus,

$$
F_{G \circ H}^{2}[a] \subseteq F_{G}^{2}[a] \cup\left[\cup_{v \in V(G) \backslash N_{G}(a)} V\left(H^{v}\right)\right]=X .
$$

Now, let $w \in X$. If $w \in F_{G}^{2}[a]$, then $w \notin N_{G}^{2}[a]$. Hence, $w \notin N_{G \circ H}^{2}[a]$. This implies that $w \in F_{G \circ H}^{2}[a]$. Suppose $w \in \cup_{v \in V(G) \backslash N_{G}(a)} V\left(H^{v}\right)$. Then there exists $v \in V(G) \backslash N_{G}(a)$ such that $w \in V\left(H^{v}\right)$. It follows that $w \neq a$ and $d_{G \circ H}(w, a) \neq 2$. Thus, $w \in F_{G \circ H}^{2}[a]$. Therefore,

$$
F_{G}^{2}[a] \cup\left[\cup_{v \in V(G) \backslash N_{G}(a)} V\left(H^{v}\right)\right] \subseteq F_{G \circ H}^{2}[a] .
$$

Case 2. Suppose $a \in V\left(H^{w}\right)$ for some $w \in V(G)$. If $x=w$, then $x \in V(G) \backslash N_{G}(w)$. Suppose $x \neq w$. If $x \in V(G)$, then $d_{G}(x, w) \neq 1$ because $d_{G \circ H}(x, a) \neq 2$. Hence, $x \in V(G) \backslash N_{G}(w)$. Suppose $x \in V\left(H^{q}\right)$ for some $q \in V(G)$. If $q=w$, then $x \in V\left(H^{w}\right)$. Since $x \neq a$ and $a \in V\left(H^{w}\right), x \in N_{H^{w}}(a)$ (otherwise, $d_{G \circ H}(a, x)=2$ ). Suppose $q \neq w$. Then $x \in V\left(H^{q}\right)$ and $q \in V(G) \backslash\{w\}$. Thus,

$$
z \in N_{H^{w}}(a) \cup\left[V(G) \backslash N_{G}(w)\right] \cup\left[\cup_{v \in V(G) \backslash\{w\}} V\left(H^{v}\right)\right]=Y .
$$


Suppose now that $p \in Y$. If $p \in N_{H^{w}}(a)$, then $d_{G \circ H}(p, a)=d_{H^{w}}(p, a)=1$. Hence, $p \in F_{G \circ H}^{2}[a]$. If $p \in V(G) \backslash N_{G}(w)$, then $d_{G \circ H}(p, w)=d_{G}(p, w) \neq 1$. Hence, $p \neq a$ and $d_{G \circ H}(a, p) \neq 2$. This implies that $p \in F_{G \circ H}^{2}[a]$. Finally, if $p \in \cup_{v \in V(G) \backslash\{w\}} V\left(H^{v}\right)$, then there exists $r \in V(G) \backslash\{w\}$ such that $p \in V\left(H^{r}\right)$. Since

$$
d_{G \circ H}(a, p)=d_{G \circ H}(a, w)+d_{G \circ H}(r, w)+d_{G \circ H}(r, p)=2+d_{G \circ H}(r, w) \geq 3,
$$

it follows that $p \in F_{G \circ H}^{2}[a]$. Therefore,

$$
N_{H^{w}}(a) \cup\left[V(G) \backslash N_{G}(w)\right] \cup\left[\cup_{v \in V(G) \backslash\{w\}} V\left(H^{v}\right)\right] \subseteq F_{G \circ H}^{2}[a] .
$$

Accordingly, the desired equality follows.

Definition 4. The lexicographic product (composition) of graphs $G$ and $H$, denoted by $G[H]$, is the graph with $V(G[H])=V(G) \times V(H)$ and $(u, v)\left(u^{\prime}, v^{\prime}\right) \in E(G[H])$ if and only if either $u u^{\prime} \in E(G)$ or $u=u^{\prime}$ and $v v^{\prime} \in E(H)$.

Theorem 6. Let $G=(V(G), E(G))$ and $H=(V(H), E(H))$ be any two graphs and let $(v, a) \in V(G[H])$. Then

$$
F_{G[H]}^{2}[(v, a)]=\left(F_{G}^{2}[v] \times V(H)\right) \cup\left(\{v\} \times F_{H}^{2}[a]\right) .
$$

Proof. Note that $(x, q) \in F_{G[H]}^{2}[(v, a)]$ if and only if $(x, q) \neq(v, a)$ and $d_{G[H]}((x, q),(v, a)) \neq 2$. Consider the following cases:

Case 1. Suppose $x=v$. Then $q \neq a$. Since

$$
d_{G[H]}((v, q),(v, a))=d_{H}(a, q) \neq 2, q \in F_{H}^{2}[a],
$$

$q \in F_{H}^{2}[a]$. Hence, $(x, q) \in\{v\} \times F_{H}^{2}[a]$.

Case 2. Suppose $x \neq v$. Then

$$
d_{G}(x, v)=d_{G[H]}((x, q),(v, a)) \neq 2 .
$$

Hence, $x \in F_{G}^{2}[v]$ and $(x, q) \in F_{G}^{2}[v] \times V(H)$. Therefore,

$$
F_{G[H]}^{2}[(v, a)] \subseteq\left(F_{G}^{2}[v] \times V(H)\right) \cup\left(\{v\} \times F_{H}^{2}[a]\right) .
$$

Next, let $(w, p) \in F_{G}^{2}[v] \times V(H)$. Then $w \in F_{G}^{2}[v]$, that is, $w \neq v$ and $d_{G}(w, v) \neq 2$. It follows that $(w, p) \neq(v, a)$ and

$$
d_{G[H]}((w, p),(v, a))=d_{G}(w, v) \neq 2 .
$$

This shows that $(w, p) \in F_{G[H]}^{2}[(v, a)]$. Hence, $F_{G}^{2}[v] \times V(H) \subseteq F_{G[H]}[(v, a)]$. Finally, let $(z, t) \in\{v\} \times F_{H}^{2}[a]$. Then $z=v$ and $t \in F_{H}^{2}[a]$. Hence, $t \neq a$ and $d_{H}(a, t) \neq 2$. Consequently, $(z, t) \neq(v, a)$ and

$$
d_{G[H]}((z, t),(v, a))=d_{H}(a, t) \neq 2,
$$

showing that $(z, t) \in F_{G[H]}^{2}[a]$. Thus, $\{v\} \times F_{H}^{2}[a] \subseteq F_{G[H]}^{2}[(v, a)]$. This establishes the desired equality. 
Definition 5. The Cartesian Product of two graphs $G_{1}$ and $G_{2}$ denoted by $G_{1} \square G_{2}$ is a graph with $V\left(G_{1} \square G_{2}\right)=V\left(G_{1}\right) \times V\left(G_{2}\right)$ and two vertices $a=\left(u_{1}, u_{2}\right)$ and $b=\left(v_{1}, v_{2}\right)$ are adjacent in $G_{1} \square G_{2}$ if and only if either $u_{1}=v_{1}$ and $u_{2} v_{2} \in E\left(G_{2}\right)$ or $u_{2}=v_{2}$ and $u_{1} v_{1} \in E\left(G_{1}\right)$.

Theorem 7. Let $K=G \square H=(V(K), E(K))$, where $G=(V(G), E(G))$ and $H=(V(H), E(H))$. Then for each $(v, a) \in V(K)$,

$$
F_{K}^{2}[(v, a)]=\left[F_{G}^{2}[v] \times\{a\}\right] \cup\left[\{v\} \times F_{H}^{2}[a]\right] \cup\left[F_{G}[v] \times V(H) \backslash\{a\}\right] \cup\left[N_{G}(v) \times F_{G}[a]\right] .
$$

Proof. Let $(v, a) \in V(K)=V(G \square H)$ and $(x, q) \in F_{K}^{2}[(v, a)]$. Then $(v, a) \neq(x, q)$ and $d_{K}((v, a),(x, q)) \neq 2$. Now, consider the following cases:

Case 1. Assume that $x=v$. Then $q \neq a$ and $d_{H}(q, a)=d_{K}((x, q),(x, a)) \neq 2$ and so, $q \in F_{H}^{2}[a]$. Hence, $(x, q) \in\{v\} \times F_{H}^{2}[a]$.

Case 2. Assume that $x \neq v$.

Subcase 1. Let $q=a$. Then $d_{G}(x, v)=d_{K}((x, q),(v, q)) \neq 2$ and thus, $x \in F_{G}^{2}[v]$. It follows that $(x, q) \in F_{G}^{2}[v] \times\{a\}$.

Subcase 2. Let $q \neq a$. Suppose that $x \in N_{G}(v)$. If $q \in N_{H}(a)$, then

$$
d_{K}((x, q),(v, a))=d_{G}(x, v)+d_{H}(q, a)=2,
$$

a contradiction. Thus, $q \in V(H) \backslash N_{H}[a]$. Hence, $(x, q) \in N_{G}(v) \times F_{G}[a]$. Suppose $x \notin N_{G}(v)$. Then $x \in F_{G}[v]$. Hence, $(x, q) \in F_{G}[v] \times V(H) \backslash\{a\}$. Therefore,

$$
F_{K}^{2}[(v, a)] \subseteq\left[F_{G}^{2}[v] \times\{a\}\right] \cup\left[\{v\} \times F_{H}^{2}[a]\right] \cup\left[F_{G}[v] \times V(H) \backslash\{a\}\right] \cup\left[N_{G}(v) \times F_{G}[a]\right] .
$$

Next, let $(v, p) \in\{v\} \times F_{H}^{2}[a]$. Then $p \neq a$ and $d_{H}(a, p) \neq 2$. Hence, $(v, p) \neq(v, a)$ and $d_{K}((v, p),(v, a))=d_{H}(a, p) \neq 2$, that is, $(v, p) \in F_{K}^{2}[(v, a)]$. If $(x, a) \in F_{G}^{2}[v] \times\{a\}$, then $x \neq v$ and $d_{G}(x, v) \neq 2$. Hence, $(x, a) \neq(v, a)$ and $d_{K}((v, a),(x, a))=d_{G}(x, v) \neq 2$, that is, $(x, a) \in F_{K}^{2}[(v, a)]$. Now, $(y, b) \in N_{G}(v) \times F_{H}[a]$ implies $d_{G}(y, v)=1$ and $d_{H}(b, a) \geq 2$. It follows that $(y, b) \neq(v, a)$ and

$$
d_{K}((y, b),(v, a))=d_{G}(y, v)+d_{H}(b, a) \geq 3 .
$$

Hence, $(y, b) \in F_{K}^{2}[(v, a)]$. Finally, $(z, t) \in\left[F_{G}[v] \times V(H) \backslash\{a\}\right]$ implies $d_{G}(z, v) \geq 2$ and $d_{H}(t, a) \geq 1$. This means that $(z, t) \neq(v, a)$ and

$$
d_{K}((z, t),(v, a))=d_{G}(z, v)+d_{H}(t, a) \geq 3 .
$$

Thus, $(z, t) \in F_{K}^{2}[(v, a)]$. Therefore,

$$
\left[F_{G}^{2}[v] \times\{a\}\right] \cup\left[\{v\} \times F_{H}^{2}[a]\right] \cup\left[F_{G}[v] \times V(H) \backslash\{a\}\right] \cup\left[N_{G}(v) \times F_{G}[a]\right] \subseteq F_{K}^{2}[(v, a)] .
$$

This establishes the desired equality. 


\section{Acknowledgements}

This research is funded by the Philippine Department of Science and TechnologyAccelerated Science and Technology Human Resource Development Program (DOSTASTHRDP) and Mindanao State University-Iligan Institute of Technology.

\section{References}

[1] S. Canoy and J. Gimeno. Which Connected Graphs Induce the Indiscrete and the Discrete Topologies? Journal of Research in Science and Engineering, 1:17-19, 2004.

[2] S. Canoy and R. Lemence. Another Look at the Topologies Induced by Graphs. Matimyas Matematika, 21:1-7, 1998.

[3] S. Canoy and R. Lemence. Topologies Induced by Some Special Graphs. Journal of Mathematics, 2:45-50, 1999.

[4] S. Canoy and C. G. Nianga. On A Finite Topological Space Induced by Hop Neighborhoods of a Graph. Advances and Applications in Discrete Mathematics, submitted.

[5] S. Diesto and S. Gervacio. Finite Topological Graphs. Journal of Research and Development, MSU-IIT, 1:76-81, 1983.

[6] R. Guerrero and S. Gervacio. Characterization of Graphs which Induce the Discrete and Indiscrete Topological Spaces. Matimyas Matematika, Special Issue, 1:11-15, 1989.

[7] F. Harary. Graph Theory. Addison-Wesley Publishing Company, USA, 1969.

[8] S. Lipschutz. General Topology, Schaum's Outline Series. McGraw Hill International Publishing Co., 1987. 